\title{
Early Results of Omitting Completion Axillary Lymph Node Dissection in Sentinel Lymph Node Metastasis-Positive Breast Cancer Patients
}

\author{
Junko Honda ${ }^{1}$, Hisashi Matsuoka ${ }^{1}$, Chieko Hirose ${ }^{1}$, Taeko Nagao ${ }^{2}$, Takahiro Yoshida ${ }^{2}$, \\ Masako Takahashi ${ }^{3}$, Issei Imoto ${ }^{4}$, Mitsunori Sasa ${ }^{3 *}$ \\ ${ }^{1}$ Department of Surgery, National Hospital Organization Higashitokushima Medical Center, \\ Tokushima, Japan \\ ${ }^{2}$ Department of Oncological and Regenerative Surgery, Institute of Health Biosciences, \\ The University of Tokushima Graduate Scholl, Tokushima, Japan \\ ${ }^{3}$ Department of Surgery, Tokushima Breast Care Clinic, Tokushima, Japan \\ ${ }^{4}$ Department of Human Genetics, Institute of Health Biosciences, The University of Tokushima Graduate School, \\ Tokushima, Japan \\ Email: *breast@mb.tcn.ne.jp
}

Received July 21, 2013; revised August 20, 2013; accepted August 28, 2013

Copyright (C) 2013 Junko Honda et al. This is an open access article distributed under the Creative Commons Attribution License, which permits unrestricted use, distribution, and reproduction in any medium, provided the original work is properly cited.

\begin{abstract}
Background: We investigated the early results of omitting completion axillary lymph node dissection (ALND) for axillary node metastasis-negative (N0), sentinel node (SN) metastasis-positive breast cancer patients. Patients and Methods: 489 patients had invasive N0 breast cancer treated without completion ALND, regardless of their SN metastasis status. Analyses included the associations between the SN metastasis status, clinicopathological findings and recurrence, between recurrence and clinicopathological findings, and recurrence-free survival. Results: 430 patients were SN biopsy (SNB)-negative, and 59 were SNB-positive. The SNB-positive patients received significantly more potent adjuvant therapy than the SNB-negative patients. Median follow-up was 3.7 years, and the axillary node recurrence was seen in 6 patients $(1.2 \%)$ and recurrence in 21 patients. The SN status showed no associations with the clinicopathological findings or recurrence. Univariate analysis showed recurrence was associated with absence of hormonal therapy, ER-negative, PgR-negative, HER2-positive or triple-negative (TNBC) disease, a tumor $\geq 2.1 \mathrm{~cm}$ and higher nuclear grade. Multivariate analysis showed recurrence was associated with absence of hormonal therapy and a tumor $\geq 2.1 \mathrm{~cm}$. Cox proportional hazards model showed recurrence was extremely early in ER-negative and TNBC patients. Conclusion: Completion ALND can be skipped in N0 breast cancer patients even if they are SNB-positive, but adjuvant therapy is essential.
\end{abstract}

Keywords: Axillary Lymph Node Dissection; Breast Cancer; Sentinel Node Biopsy

\section{Introduction}

Sentinel lymph node biopsy (SNB) has been used as a standard procedure for the management of early breast cancer patients who have no clinical axillary lymph node metastasis (N0) [1,2]. If the SN is negative, axillary dissection (ALND) can be omitted, but if the $\mathrm{SN}$ is positive for metastasis, completion ALND is generally warranted [3]. However, after release of the results of the American College of Surgeon's Oncology Group (ACOSOG) Z0011 randomized clinical trial [4], the National Com-

\footnotetext{
${ }^{*}$ Corresponding author.
}

prehensive Cancer Network's (NCCN) Practice Guidelines issued in 2012 recommended avoidance of completion ALND as an option in selected SNB-positive cases [5].

Since March 2003, which predates that NCCN recommendation, we have been omitting the completion ALND in N0 patients even if they are SNB-positive. The scientific basis for that policy is as follows: 1) First, in the case of axillary node micrometastasis, it was reported that the incidence of axillary recurrence is low even when completion ALND is not performed, and the survival results do not differ between the dissected and 
non-dissected patient groups [6-8], and 2) then, even in the case of overt axillary metastasis, it was shown that rescue surgery was feasible and there was no effect on survival [6-8]. Therefore, since 2003 we obtain written informed consent and then skip ALND in selected SNB-positive cases. To date, the median follow-up period for our patients has been over 3.7 years, and we think that our data are of value for assessing lymph node recurrence. Accordingly, we report here the results of our comparative analysis of SNB-positive patients who did not undergo completion ALND and SNB-negative patients. We compared the two patient groups in regard to the clinicopathological findings and recurrence, tested for associations between recurrence and the clinicopathological findings, and also analyzed the recurrence-free survival.

\section{Patients and Methods}

We identified the SN using CT-lymphography (CTLG) combined with dye-staining $[9,10]$ because our institution is not equipped with RI facilities. Clinicopathological studies to determine the metastatic status of SNs were performed on 2-mm sections of hematoxylin-eosin (HE)stained surgical permanent specimens. SNB-positive cases were treated by one of two methods chosen in accordance with each patient's expressed desire after written informed consent had been obtained. That is, when the SNB was positive for metastasis following the surgery, the patient was allowed to choose one of two treatment options: 1) performance of completion ALND, followed by performance of adjuvant therapy, or 2) performance of appropriate adjuvant therapy, including axillary radiation therapy, with repeat performance of dissection at the time of any overt recurrence. For patients whose SNB was negative for metastasis, adjuvant therapy was decided on the basis of the subtype classification of the primary lesion. This study was carried out after its design had been approved by the Ethics Committee of National Hospital Organization Higashi-Tokushima Medical Center.

From March 2003 through September 2012, our hospital treated 663 primary breast cancer patients by performing axillary surgery that was limited to SNB. This study enrolled 489 of those patients who had invasive ductal carcinoma, while 46 patients who had undergone preoperative chemotherapy, 102 patients with ductal carcinoma in situ and 26 patients with a special pathological subtype of disease were excluded.

Recurrent breast cancer can have various presentations: axillary lymph node recurrence, recurrence in other lymph nodes (i.e., the ipsilateral supraclavicular, subclavicular and internal mammary nodes), distant recurrence, recurrence in the ipsilateral breast and recurrence in the contralateral breast. For this study, contralateral breast cancer recurrence was considered to be development of a new lesion and thus excluded from the category of "recurrence".

The clinicopathological findings were analyzed for associations with the SNB status, i.e., positive or negative for metastasis. Recurrence was also analyzed for association with the status of SN metastasis. Moreover, for all recurrent events (excluding contralateral breast cancer recurrence), the status of recurrence and the clinicopathological findings were analyzed for associations and recurrence-free survival was also analyzed.

For statistical analyses of the data, Fisher's exact test was used to analyze for associations between the status of SN metastasis (positive vs. negative) and the clinicopathological findings, including recurrence. Clinicopathological characteristics that showed possible association with recurrence in univariate analysis, were analyzed by multivariate analysis using logistic regression analysis. Recurrence-free survival was analyzed using the Cox proportional hazards model, taking into account several covariates. Statistical tests provided two-sided $\mathrm{p}$ values, and a significance level of $\mathrm{p}<0.05$ was used. Statistical analyses were carried out using the $\mathrm{R}$ statistical environment version 2.13.0 (http://www.r-project.org/).

\section{Results}

\subsection{Demographic Data for the 489 Enrolled Patients and Comparison of Pathological Findings in SNB-Negative Patients and SNB-Positive Patients}

The age range was $29-85$ years (median: 55 y), with $33 \%$ being $\leq 49$ yo and $67 \%$ being $\geq 50$ yo. The number of biopsied lymph nodes per patient was $1-3$, with a mean of 1.7. Receptor analyses indicated that the rates of ERpositive, PgR-positive, HER2-positive and triple-negative (TNBC) patients were $80 \%, 70 \%, 6 \%$ and $11 \%$, respectively. The tumor size was $\leq 2 \mathrm{~cm}$ in $86 \%$ of the patients, while $62 \%$ were nuclear grade 1 . The surgical procedure was breast-conserving therapy in $91 \%$ of the patients. SNB-negative patients numbered $430(87.9 \%)$, while 59 patients (12.1\%) were SNB-positive. Table 1 shows that comparative analysis of the clinicopathological characteristics as a function of the SNB-negative/-positive status found no statistically significant differences.

\subsection{Comparison of Adjuvant Therapy in SNB-Negative and SNB-Positive Patients}

Table 2 shows the results of comparison of the types of adjuvant therapy administered to the SNB-negative and SNB-positive patients. Axillary radiation was administered to 18 of the 59 SNB-positive patients, but to none of the 430 SNB-negative patients. Chemotherapy was 
administered to 34 of the 59 SNB-positive patients and to 38 of the 430 SNB-negative patients. Finally, hormonal therapy was administered to a majority of the patients, regardless of the status of SN metastasis. It is thus clear that the SNB-positive patients received more potent adjuvant therapies, i.e., axillary radiation and chemotherapy, compared with the SNB-negative patients.

\subsection{Events of Recurrence}

Table 3 compiles the data on the events of recurrence (some overlapping exists). The median follow-up period was 3.7 years, and recurrence was documented in 21 patients. The types of recurrence were distant metastatic events in 10 patients $(2.0 \%)$, axillary node recurrence in 6 patients $(1.2 \%)$, recurrence in other nodes in 3 patients $(0.6 \%)$ and recurrence in the ipsilateral breast in 7 patients $(1.4 \%)$. No recurrence was seen in 468 patients.

Table 1. Comparison of clinicopathological findings in SNB-negative patients and SNB-positive patients (excluding data for unknown cases).

\begin{tabular}{|c|c|c|c|}
\hline & $\begin{array}{l}\text { No. of } \\
\text { SNB-negative } \\
\text { patients } \\
(\mathrm{n}=430)\end{array}$ & $\begin{array}{l}\text { No. of } \\
\text { SNB-positive } \\
\text { patients } \\
(\mathrm{n}=59)\end{array}$ & $\mathrm{p}$ value \\
\hline \multicolumn{4}{|l|}{ ER } \\
\hline Negative & 73 & 6 & \multirow{2}{*}{ NS } \\
\hline Positive & 342 & 52 & \\
\hline \multicolumn{4}{|l|}{$\mathrm{PgR}$} \\
\hline Negative & 118 & 10 & \multirow{2}{*}{ NS } \\
\hline Positive & 297 & 48 & \\
\hline \multicolumn{4}{|l|}{ HER2 } \\
\hline Negative & 369 & 54 & \multirow{2}{*}{ NS } \\
\hline Positive $^{*}$ & 28 & 2 & \\
\hline \multicolumn{4}{|l|}{ Triple-negative } \\
\hline No & 350 & 51 & \multirow{2}{*}{ NS } \\
\hline Yes & 47 & 5 & \\
\hline \multicolumn{4}{|l|}{ Tumor size $(\mathrm{cm})$} \\
\hline$\leq 2$ & 375 & 47 & \multirow{2}{*}{ NS } \\
\hline $2.1-5$ & 55 & 12 & \\
\hline \multicolumn{4}{|l|}{ Nuclear grade } \\
\hline 1 & 270 & 34 & \multirow{3}{*}{ NS } \\
\hline 2 & 66 & 15 & \\
\hline 3 & 53 & 8 & \\
\hline \multicolumn{4}{|l|}{ Surgical procedure } \\
\hline $\begin{array}{c}\text { Breast-conserving } \\
\text { therapy }\end{array}$ & 390 & 56 & \multirow{2}{*}{ NS } \\
\hline Mastectomy & 40 & 3 & \\
\hline
\end{tabular}

${ }^{*}$ Score of $3^{+}$in immunohistochemistry or fluorescence in situ hybridization-positive. ER: estrogen receptor; PgR: progesterone receptor; HER2: human epidermal growth factor receptor 2; NS: not significant.
However, after exclusion of 17 patients with contralateral breast events, 451 patients were considered to have had no events of recurrence and were used for the subsequent analyses.

\subsection{Comparison of Events of Recurrence in SNB-Negative and SNB-Positive Patients}

Table 4 shows the comparison of the recurrences in the SNB-negative and SNB-positive patients. No statistically

Table 2. Comparison of adjuvant therapy SNB-negative patients and SNB-positive patients.

\begin{tabular}{cccccccc}
\hline & \multicolumn{3}{c}{ Axillary radiation } & \multicolumn{2}{c}{ Chemotherapy } & \multicolumn{2}{l}{ Hormonal therapy } \\
\cline { 2 - 7 } & No & Yes & No & Yes & No & Yes \\
\hline $\begin{array}{c}\text { SNB-negative } \\
\text { patients }\end{array}$ & 430 & 0 & 392 & 38 & 96 & 334 \\
$\begin{array}{c}\text { SNB-positive } \\
\text { patients } \\
\text { p value }\end{array}$ & 41 & 18 & 25 & 34 & 15 & 44 \\
\hline
\end{tabular}

NS: not significant.

Table 3. Events of recurrence (includes some overlapping).

\begin{tabular}{ccc}
\hline & No. of patients $(\mathrm{n}=489)$ & $\%$ \\
\hline No event & 451 & 92.2 \\
Locoregional events & 14 & 2.9 \\
Tumor in breast & 7 & 1.4 \\
Axillary node recurrence & 6 & 1.2 \\
Other node recurrence & 3 & 0.6 \\
Distant metastasis events & 10 & 2.0 \\
Contralateral breast events & 17 & 3.5 \\
\hline
\end{tabular}

*Other nodes include ipsilateral supraclavicular, subclavicular and internal mammary nodes.

Table 4. Comparison of events of recurrence in SNB-negative and SNB-positive patients.

\begin{tabular}{|c|c|c|c|}
\hline & $\begin{array}{c}\text { No. of } \\
\text { SNB-negative } \\
\text { patients }\end{array}$ & $\begin{array}{c}\text { No. of } \\
\text { SNB-positive } \\
\text { patients }\end{array}$ & $\mathrm{p}$ value \\
\hline \multicolumn{4}{|c|}{ Distant metastasis } \\
\hline Negative & 421 & 58 & \multirow{2}{*}{ NS } \\
\hline Positive & 9 & 1 & \\
\hline \multicolumn{4}{|c|}{ Axillary node recurrence } \\
\hline Negative & 426 & 57 & \multirow{2}{*}{ NS } \\
\hline Positive & 4 & 2 & \\
\hline \multicolumn{4}{|c|}{ Other node recurrence } \\
\hline Negative & 427 & 59 & \multirow{2}{*}{ NS } \\
\hline Positive & 3 & 0 & \\
\hline \multicolumn{4}{|c|}{ Tumor in breast } \\
\hline Negative & 423 & 59 & \multirow{2}{*}{ NS } \\
\hline Positive & 7 & 0 & \\
\hline
\end{tabular}

NS: not significant. 
significant associations were found between any of the types of recurrences and the status of SN metastasis.

\subsection{Clinical and Pathological Findings as a Function of the Recurrence Status}

Table 5 shows the associations between the status of recurrences and the clinicopathological findings evaluated by univariate analysis using Fisher's exact test and by multivariate analysis using logistic regression analysis. The univariate analysis found statistically significant associations between recurrence and each of the following clinicopathological findings: no hormonal therapy,
ER-negative, PgR-negative, HER2-positive, TNBC, a tumor size of $\geq 2.1 \mathrm{~cm}$ and a higher nuclear grade. The multivariate analysis found that recurrence was significantly more frequent in patients who had not undergone hormonal therapy or had a tumor size of $\geq 2.1 \mathrm{~cm}$.

\subsection{Multivariate Analysis of All Recurrence-Free Survival}

Table 6 shows the results of multivariate analysis performed using the Cox proportional hazards model to examine for associations between the recurrence-free survival and the clinicopathological findings. The results

Table 5. Clinicopathological findings as a function of the recurrence status (excluding data for unknown cases).

\begin{tabular}{|c|c|c|c|c|}
\hline \multicolumn{2}{|c|}{$<$ Univariate analysis $>$} & No recurrence & Recurrence $^{*}$ & $\mathrm{p}$ value \\
\hline \multirow{2}{*}{ Age } & $\geq 50$ & 315 & 15 & \multirow{2}{*}{ NS } \\
\hline & $<50$ & 153 & 6 & \\
\hline \multirow{2}{*}{ Axillary radiation therapy } & No & 450 & 21 & \multirow{2}{*}{ NS } \\
\hline & Yes & 18 & 0 & \\
\hline \multirow{2}{*}{ Chemotherapy } & No & 401 & 16 & \multirow{2}{*}{ NS } \\
\hline & Yes & 67 & 5 & \\
\hline \multirow{2}{*}{ Hormonal therapy } & No & 99 & 12 & \multirow{2}{*}{0.0005} \\
\hline & Yes & 369 & 9 & \\
\hline \multirow{2}{*}{ ER } & Negative & 70 & 9 & \multirow{2}{*}{0.00347} \\
\hline & Positive & 382 & 12 & \\
\hline \multirow{2}{*}{$\operatorname{PgR}$} & Negative & 117 & 11 & \multirow{2}{*}{0.0115} \\
\hline & Positive & 335 & 10 & \\
\hline \multirow{2}{*}{ HER2 } & Negative & 406 & 17 & \multirow{2}{*}{0.0424} \\
\hline & Positive $^{* *}$ & 26 & 4 & \\
\hline \multirow{2}{*}{ Triple-negative } & No & 387 & 14 & \multirow{2}{*}{0.00574} \\
\hline & Yes & 45 & 7 & \\
\hline \multirow{2}{*}{ Tumor size $(\mathrm{cm})$} & $\leq 2$ & 408 & 14 & \multirow{2}{*}{0.0159} \\
\hline & $2.1-5$ & 60 & 7 & \\
\hline \multirow{3}{*}{ Nuclear grade } & 1 & 298 & 6 & \multirow{3}{*}{0.00424} \\
\hline & 2 & 76 & 5 & \\
\hline & 3 & 55 & 6 & \\
\hline \multirow{2}{*}{ Surgical procedure } & Breast-conserving therapy & 427 & 19 & \multirow{2}{*}{ NS } \\
\hline & Mastectomy & 41 & 2 & \\
\hline \multirow{2}{*}{ Sentinel node biopsy (SNB) } & Negative & 411 & 19 & \multirow{2}{*}{ NS } \\
\hline & Positive & 57 & 2 & \\
\hline \multicolumn{2}{|c|}{$<$ Multivariate analysis $>$} & Odds ratio ( $95 \%$ Confidence Interval) & \multicolumn{2}{|c|}{$\mathrm{p}$ value } \\
\hline \multirow{2}{*}{ Hormonal therapy } & No & $4.785(1.727-13.245)$ & \multirow{2}{*}{\multicolumn{2}{|c|}{0.00261}} \\
\hline & Yes & 1.00 & & \\
\hline \multirow{2}{*}{ Tumor size } & $\leq 2$ & 1.00 & \multirow{2}{*}{\multicolumn{2}{|c|}{0.0163}} \\
\hline & $2.1-5$ & $3.590(1.260-10.200)$ & & \\
\hline
\end{tabular}

${ }^{*}$ Recurrence includes locoregional events and distant metastasis events. ${ }^{* *}$ Score of $3^{+}$in immunohistochemistry or fluorescence in situ hybridization positive. ER: estrogen receptor; PgR: progesterone receptor; HER2: human epidermal growth factor receptor 2; NS: not significant. 
Table 6. Multivariate analysis of all recurrence-free survival.

\begin{tabular}{|c|c|c|}
\hline & Odds ratio ( $95 \%$ Confidence Interval) & $\mathrm{p}$ value \\
\hline \multicolumn{3}{|c|}{ Hormonal therapy } \\
\hline No & $8.13(2.179-30.30)$ & \multirow{2}{*}{0.0018} \\
\hline \multirow[t]{2}{*}{ Yes } & 1.00 & \\
\hline & ER & \multirow{4}{*}{0.0025} \\
\hline Negative & $58.82(4.167-1000)$ & \\
\hline Positive & 1.00 & \\
\hline & HER2 & \\
\hline Negative & 1.00 & \multirow{2}{*}{0.0013} \\
\hline Positive ${ }^{*}$ & $13.75(2.784-67.920)$ & \\
\hline \multicolumn{3}{|c|}{ Triple-negative } \\
\hline No & 1.00 & \multirow{2}{*}{0.0053} \\
\hline Yes & $46.12(3.112-683.500)$ & \\
\hline \multicolumn{3}{|c|}{ Tumor size $(\mathrm{cm})$} \\
\hline$\leq 2$ & 1.00 & \multirow{2}{*}{0.0042} \\
\hline $2.1-5$ & $4.31(1.585-11.720)$ & \\
\hline
\end{tabular}

${ }^{*}$ Score of $3^{+}$in immunohistochemistry or fluorescence in situ hybridization positive. ER: estrogen receptor; PgR: progesterone receptor; HER2: human epidermal growth factor receptor 2 .

showed that recurrence took place significantly early in patients who had not undergone hormonal therapy, were ER-negative, HER2-positive or TNBC, or had a tumor size of $\geq 2.1 \mathrm{~cm}$. In particular, recurrence was extremely early in ER-negative (odds ratio of 58.82) and TNBC (odds ratio of 46.12) patients.

\section{Discussion}

A randomized clinical trial was started in 2001 with the aim of demonstrating the non-inferiority of non-performance of completion ALND in breast cancer patients with an SN positive for metastasis (IBCSG Trial 23-01) [11]. No significant differences were found in terms of the axillary recurrence rate, recurrence-free survival rate or overall survival rate. The Z0011 randomized trial also reported the appropriateness of avoidance of completion ALND even in T1-2 N0 patients who were SNB-positive [4]. The cohort research report released by the U.S.A. National Cancer Data Base also found that although ALND was skipped for about $20 \%$ of SNB-positive cases in actual clinical settings, the 5-year axillary recurrence rate was only $1 \%$ [12]. Reflecting those findings, the NCCN Practice Guidelines issued in 2012 states that avoidance of completion ALND is an option for SNBpositive breast cancer patients who satisfy the criteria set forth in the Z0011 study [5].

Since 2003, with approval from our Ethics Committee, we have been obtaining written informed consent and then omitting ALND in N0 patients even if they are
SNB-positive. The median follow-up period for our patients has reached 3.7 years. Since it was reported that most events of axillary recurrence in $\mathrm{N} 0$ patients without completion ALND occur within 3 years after surgery $[6,13]$, we felt that our present data were at least good for the relationship between that therapeutic approach and disease recurrence. Our analysis of those data showed a $1.2 \%$ incidence rate for axillary recurrence in N0 breast cancer patients who underwent only SNB. That recurrence rate is comparable to the rates reported by others $[12,14-16]$, and we think that it sufficiently guarantees safety in regard to axillary recurrence. In addition, we found no statistically significant differences between the status of SN metastasis and the site of recurrence, although the axillary recurrence rate was slightly higher in patients who had been SNB-positive. All other sites showed no difference. Our present results provide further evidence of the reliability of the Z0011 study results [4] and the NCCN Practice Guidelines recommendation [5]. Taking into consideration the facts that rescue surgery can still be carried out in the case of axillary recurrence and that suitable adjuvant therapy is administered to N0 patients even if they are SNB-positive, we think that it is possible to omit completion ALND in these patients.

Next, we analyzed for associations between recurrence and the patients' background factors. Most of the patients in our present series received adjuvant therapies based on their disease subtype. In addition, with a median follow-up of only 3.7 years, this study can be considered short-term. Our univariate analysis found that, with regard to adjuvant treatment, recurrence was significantly more frequent in patients who had not undergone hormonal therapy. This finding seems to mean that patients who did not receive hormonal therapy were negative for both ER and PgR. With regard to the malignancy of breast cancer, our results showed significantly higher incidences of recurrence in patients who were ER-negative, PgR-negative, HER2-positive or TNBC, or had a larger tumor size or a higher nuclear grade. In addition, our multivariate analysis found that recurrence was significantly more frequent in patients who had not undergone hormonal therapy or had a larger tumor size. That is, when adjuvant therapy was administered to N0 patients in consideration of their disease subtype, negative status for both ER and PgR and the larger tumor size can be considered independent poorer predictors of recurrence in the early postoperative stage. There is a possibility that the tumor size became an independent predictor of recurrence, exactly because it had not been taken into account when deciding the adjuvant therapy for our patients. Moreover, it is possible that the cohort negative for both ER and PgR (which includes TNBC) includes a population whose disease has an especially high degree of malignancy. Our data also indicates that recurrence 
occurs very early in ER-negative and TNBC patients. It has been proposed that various types are included in TNBC [17], and it will be necessary to identify the highly-malignant subtypes having various surrogate markers and establish appropriate therapeutic approaches for them.

We selected chemotherapy plus hormonal therapy for SNB-positive patients even when they had the luminal A subtype. At present, it is thought that chemotherapy is not necessary for the luminal A subtype if the number of metastatic lymph nodes is small [18]. Accordingly, it is possible that we administered unnecessary chemotherapy for our SNB-positive patient group. This warrants further investigation in the future.

The small number of analyzed cases and the rather short duration of follow-up are other limitations of this study. Also, this study analyzed the clinicopathological characteristics of invasive N0 breast cancer patients for association with recurrence, but did not analyze with regard to survival. In the future, we are willing to perform further analyses, including of the relationship between omitting completion ALND and the survival results, in a larger number of patients over a longer follow-up period.

\section{Conclusion}

This study found that completion ALND could be omitted for N0 breast cancer patients even if they were SNB-positive, and provided that appropriate adjuvant therapy was administered. Also, when suitable adjuvant therapy was administered to N0 patients, even during the course of short-term observation recurrence was significantly more common in patients who were hormone recaptor-negative or had a large tumor, those results were shown to be unrelated to whether or not there had been $\mathrm{SN}$ metastasis.

\section{REFERENCES}

[1] T. Kim, A. E. Giuliano and G. H. Lyman, "Lymphatic Mapping and Sentinel Lymph Node Biopsy in Early-Stage Breast Carcinoma: A Metaanalysis," Cancer, Vol. 106, No. 1, 2006, pp. 4-16. http://dx.doi.org/10.1002/cncr.21568

[2] M. R. Kell, J. P. Burke, M. Barry and M. Morrow, "Outcome of Axillary Staging in Early Breast Cancer: A Meta-Analysis," Breast Cancer Research and Treatment, Vol. 120, No. 2, 2010, pp. 441-447. http://dx.doi.org/10.1007/s10549-009-0705-6

[3] G. H. Lyman, A. E. Giuliano, M. R. Somerfield, A. B. 3rd Benson, D. C. Bodurka, H. J. Burstein, A. V. Cochran, H. S. 3rd Cody, S. B. Edge, S. Galper, J. A. Hayman, T. Y. Kim, C. L. Perkins, D. A. Podoloff, V. H. Sivasubramaniam, R. R. Turner, R. Wahl, D. L. Weaver, A. C. Wolff and E. P. Winer, "American Society of Clinical
Oncology: American Society of Clinical Oncology Guideline Recommendations for Sentinel Lymph Node Biopsy in Early-Stage Breast Cancer," Journal of Clinical Oncology, Vol. 23, No. 30, 2005, pp. 7703-7720. http://dx.doi.org/10.1200/JCO.2005.08.001

[4] A. E. Giuliano, K. K. Hunt, K. V. Ballman, P. D. Beitsch, P. W. Whitworth, P. W. Blumencranz, A. M. Leitch, S. Saha, L. M. McCall and M. Morrow, "Axillary Dissection vs No Axillary Dissection in Women with Invasive Breast Cancer and Sentinel Node Metastasis: A Randomized Clinical Trial," Journal of American Medical Association, Vol. 305, No. 6, 2011, pp. 569-575. http://dx.doi.org/10.1001/jama.2011.90

[5] "National Comprehensive Cancer Network, Clinical Practice Guidelines in Oncology. Breast Cancer. ver. 1,” 2013. http://www.nccn.org/index.asp

[6] B. Fisher, J. H. Jeong, S. Anderson, J. Bryant, E. R. Fisher and N. Wolmark, "Twenty-Five-Year Follow-Up of a Randomized Trial Comparing Radical Mastectomy, Total Mastectomy, and Total Mastectomy Followed by Irradiation," New England Journal of Medicine, Vol. 347, No. 8, 2002, pp. 567-575. http://dx.doi.org/10.1056/NEJMoa020128

[7] J. M. Guenther, N. M. Hansen, L. A. DiFronzo, A. E. Giuliano, J. C. Collins, B. L. Grube and T. X. O'Connell, "Axillary Dissection Is Not Required for All Patients with Breast Cancer and Positive Sentinel Nodes," Archives of Surgery, Vol. 138, No. 1, 2003, pp. 52-56. http://dx.doi.org/10.1001/archsurg.138.1.52

[8] J. S. Fant, M. D. Grant, S. M. Knox, S. A. Livingston, K. Ridl, R. C. Jones and J. A. Kuhn, "Preliminary Outcome Analysis in Patients with Breast Cancer and a Positive Sentinel Lymph Node Who Declined Axillary Dissection," Annals of Surgical Oncology, Vol. 10, No. 2, 2003, pp. 126-130. http://dx.doi.org/10.1245/ASO.2003.04.022

[9] M. Minato, C. Hirose, M. Sasa, H. Nishitani, Y. Hirose and T. Morimoto, " 3-Dimensional Computed Tomography Lymphography-Guided Identification of Sentinel Lymph Nodes in Breast Cancer Patients Using Subcutaneous Injection of Nonionic Contrast Medium: A Clinical Trial," Journal of Computer Assisted Tomography, Vol. 28, No. 1, 2004, pp. 46-51. http://dx.doi.org/10.1097/00004728-200401000-00007

[10] M. Takahashi, M. Sasa, C. Hirose, S. Hisaoka, M. Taki, T. Hirose and Y. Bando, "Clinical Efficacy and Problems with CT Lymphography in Identifying the Sentinel Node in Breast Cancer," World Journal of Surgical Oncology, Vol. 6, 2008, p. 57. http://dx.doi.org/10.1186/1477-7819-6-57

[11] V. Galimberti, E. Botteri, C. Chifu, O. Gentilini, A. Luini, M. Intra, P. Baratella, M. Sargenti, S. Zurrida, P. Veronesi, N. Rotmensz, G. Viale, A. Sonzogni, M. Colleoni and U. Veronesi, "Can We Avoid Axillary Dissection in the Micrometastatic Sentinel Node in Breast Cancer?" Breast Cancer Research and Treatment, Vol. 131, No. 3, 2012, pp. 819-825. http://dx.doi.org/10.1007/s10549-011-1486-2

[12] K. Y. Bilimoria, D. J. Bentrem, N. M. Hansen, K. P. Bethke, A. W. Rademaker, C. Y. Ko, D. P. Winchester and D. J. Winchester, "Comparison of Sentinel Lymph 
Node Biopsy Alone and Completion Axillary Lymph Node Dissection for Node-Positive Breast Cancer," Journal of Clinical Oncology, Vol. 27, No. 18, 2009, pp. 2946-2953. http://dx.doi.org/10.1200/JCO.2008.19.5750

[13] M. Greco, R. Agresti, N. Cascinelli, P. Casalini, R. Giovanazzi, A. Maucione, G. Tomasic, C. Ferraris, M. Ammatuna, S. Pilotti and S. Menard, "Breast Cancer Patients treated without Axillary Surgery: Clinical Implications and Biologic Analysis," Annals of Surgery, Vol. 232, No. 1, 2000, pp. 1-7. http://dx.doi.org/10.1097/00000658-200007000-00001

[14] A. M. Naik, J. Fey, M. Gemignani, A. Heerdt, L. Montgomery, J. Petrek, E. Port, V. Sacchini, L. Sclafani, K. VanZee, R. Wagman, P. I. Borgen and H. S. Cody 3rd, "The Risk of Axillary Relapse after Sentinel Lymph Node Biopsy for Breast Cancer Is Comparable with That of Axillary Lymph Node Dissection: A Follow-Up Study of 4008 Procedures," Annals of Surgery, Vol. 240, No. 3, 2004, pp. 462-468.

http://dx.doi.org/10.1097/01.sla.0000137130.23530.19

[15] J. Park, J. V. Fey, A. M. Naik, P. I. Borgen, K. J. Van Zee and H. S. Cody 3rd, "A Declining Rate of Completion
Axillary Dissection in Sentinel Lymph Node-Positive Breast Cancer Patients Is Associated with the Use of a Multivariate Nomogram," Annals of Surgery, Vol. 245, No. 3, 2007, pp. 462-468. http://dx.doi.org/10.1097/01.sla.0000250439.86020.85

[16] L. Spiguel, K. Yao, D. J. Winchester, A. Gorchow, H. Du, S. F. Sener, B. Martz, M. Turk, E. Barrera and D. P. Winchester, "Sentinel Node Biopsy Alone for NodePositive Breast Cancer: 12-Year Experience at a Single Institution," Journal of the American College of Surgeons, Vol. 213, No. 1, 2011, pp. 122-128. http://dx.doi.org/10.1016/j.jamcollsurg.2011.03.034

[17] H. Joensuu and J. Gligorov, "Adjuvant Treatments for Triple-Negative Breast Cancers," Annals of Oncology, Vol. 23, Suppl. 6, 2012, pp. vi40-vi45. http://dx.doi.org/10.1093/annonc/mds194

[18] A. S. Coates, M. Colleoni and A. Goldhirsch, "Is Adjuvant Chemotherapy Useful for Women with Luminal a Breast Cancer?" Journal of Clinical Oncology, Vol. 30, No. 12, 2012, pp. 1260-1263. http://dx.doi.org/10.1200/JCO.2011.37.7879 